University of Texas at El Paso

ScholarWorks@UTEP

4-2002

\title{
On Efficient Representation of Expert Knowledge by Fuzzy Logic: Towards an Optimal Combination of Granularity and Higher-Order Approaches
}

\author{
Hung T. Nguyen \\ Vladik Kreinovich \\ The University of Texas at El Paso, vladik@utep.edu
}

Follow this and additional works at: https://scholarworks.utep.edu/cs_techrep

Part of the Computer Engineering Commons

Comments:

UTEP-CS-01-24b.

Published in: Ajith Abraham, Lakhmi Jain, and Janusz Kacprzyk (eds.), Recent Advances in Intelligent Paradigms and Applications, Springer-Verlag, 2002, pp. 107-132.

\section{Recommended Citation}

Nguyen, Hung T. and Kreinovich, Vladik, "On Efficient Representation of Expert Knowledge by Fuzzy Logic: Towards an Optimal Combination of Granularity and Higher-Order Approaches" (2002). Departmental Technical Reports (CS). 411.

https://scholarworks.utep.edu/cs_techrep/411

This Article is brought to you for free and open access by the Computer Science at ScholarWorks@UTEP. It has been accepted for inclusion in Departmental Technical Reports (CS) by an authorized administrator of ScholarWorks@UTEP.For more information, please contact Iweber@utep.edu. 


\title{
On Efficient Representation of Expert Knowledge by Fuzzy Logic: Towards an Optimal Combination of Granularity and Higher-Order Approaches
}

\author{
Hung T. Nguyen ${ }^{1}$ and Vladik Kreinovich ${ }^{2}$ \\ 1 Department of Mathematical Sciences, New Mexico State University, \\ Las Cruces, NM 88003, USA, email hunguyen@nmsu.edu \\ 2 Department of Computer Science, University of Texas at El Paso, \\ El Paso, TX 79968, USA, email vladik@cs.utep.edu
}

\begin{abstract}
A natural approach to designing an intelligent system is to incorporate expert knowledge into this system. One of the main approaches to translating this knowledge into computer-understandable terms is the approach of fuzzy logic. It has led to many successful applications, but in several aspects, the resulting computer representation is somewhat different from the original expert meaning. Two related approaches have been used to make fuzzy logic more adequate in representing expert reasoning: granularity and higher-order approaches. Each approach is successful in some applications where the other approach did not succeed so well; it is therefore desirable to combine these two approaches. This idea of combining the two approaches is very natural, but so far, it has led to few successful practical applications. In this chapter, we provide results aimed at finding a better (ideally optimal) way of combining these approaches.
\end{abstract}

Keywords: expert knowledge, fuzzy logic, granularity, higher-order approach

\section{Introduction: Fuzzy Logic, Granularity, and Higher Order Approaches}

Why fuzzy logic. A natural approach to designing an intelligent system is to incorporate expert knowledge into this system.

Experts are often not $100 \%$ certain in the statements they make; therefore, in the design of knowledge-based systems, it is desirable to take this uncertainty into consideration. Usually, this uncertainty is described by a number from the interval $[0,1]$; this number is called subjective probability, degree of certainty, etc. (see, e.g., [50]). Situations when an expert is $100 \%$ sure that a statement $S$ is true are describe by the subjective probability $d(S)=1$; situations when an expert is $100 \%$ sure that a statement is false are described by the subjective probability $d(S)=0$; situations when an expert is not sure about a statement are described by intermediate values $d(S) \in(0,1)$.

The use of values from the interval $[0,1]$ to describe the expert's certainty is the main idea behind fuzzy logic. Fuzzy logic, introduced by L. Zadeh 
is 1965 [55], is currently one of the main approaches to translating expert knowledge into computer-understandable terms; see, e.g., [19,45].

Beyond [0, 1]-based fuzzy logic. The [0,1]-based approach has led to many successful applications. However, the [0,1]-based fuzzy logic is, by itself, only an approximation to the actual human reasoning about uncertainty.

Indeed, how can we describe the expert's degree of confidence $d(S)$ in a certain statement $S$ ? A natural way to determine this degree is, e.g., to ask an expert to estimate his degree of confidence on a scale from 0 to 10 . If he selects 8 , then we take $d(S)=8 / 10$.

To get a more accurate result, we can then ask the same expert to estimate his degree of confidence on a finer scale, e.g., from 0 to 100, etc. For example, if an expert selects 81 , we will take $d(S)=81 / 100=0.81$. If we want an even more accurate estimate, we can ask the expert to estimate his degree of confidence on an even finer scale, etc.

The problem with this approach is that experts cannot describe their degrees of too fine scales. For example, an expert can point to 8 on a scale from 0 to 10 , but this same expert will hardly be able to pinpoint a value on a scale from 0 to 100 .

So, to attain a more adequate description of human reasoning, we must modify the traditional $[0,1]$-based fuzzy logic. Two types of modifications have been proposed.

Granularity. One modification - proposed by Zadeh in [56] - is to use the granularity of expert estimates. The simplest way of using this granularity is to take the finest (finite) scale which an expert can still use, and take the values corresponding to this scale as the desired degrees of confidence.

For example, if an expert can meaningfully describe his or her degree of confidence on a scale from 0 to 10 , but not on a scale from 0 to 11 , then the scale 0 to 10 is the finest scale which an expert can still use.

This approach leads to a finite-valued fuzzy logic, in which the set of truth values $V$ is finite. For example, if the finest scale is a scale from 0 to 10 , then possible degrees of confidence are $0 / 10,1 / 10, \ldots, 1=10 / 10$ - overall, 11 possible degrees. This approach has been successfully used in practice; see, e.g., $[1,13,28,39,47]$.

Interval-valued approach. Another modification - also originally proposed by Zadeh - is to describe the expert's degree of confidence by an interval from $[0,1]$. For example, if an expert estimates his degree of confidence by a value 8 on a 0 to 10 scale, then the only thing that we know about the expert's degree of confidence is that it is closer to $0.8(8 / 10)$ than to 0.7 or to 0.9 , i.e., that it belongs to the interval $[0.75,0.85]$.

So, within this second modification, a natural way of describing degrees of confidence more adequately is to use intervals $\mathbf{a}=\left[a^{-}, a^{+}\right]$instead of real numbers. In this representation, real numbers can be viewed as particular degenerate - cases of intervals $[a, a]$. The Zadeh's idea of using intervals have 
been further developed by Bandler and Kohout [2], Türkşen [52], and others; for a recent survey, see, e.g., [32,44].

From interval-valued to more general second order approach. In the interval-valued fuzzy approach, to describe each degree of confidence, we must describe two real numbers: the lower endpoint and the upper endpoint of the corresponding "confidence interval".

We can go one step further and take into consideration that the endpoints of the corresponding interval are also not precisely known. Thus, each of these endpoints is, in actuality, an interval itself. So, to describe a degree of confidence, we now need four real numbers: two to describe the lower endpoint, and two to describe the upper one.

This modified interval approach is also only an approximation to real reasoning. Indeed, in this approach, we assume that while an expert cannot describe his degree of certainty $d$ in a statement $A$ by a single number, he is, nevertheless, perfectly able, given a statement $A$ and a degree $d$, to tell whether $d$ is a possible degree of certainty. In reality, if you ask the expert whether his degree of certainty about a certain statement $A$ can be described by a certain number $d$ (e.g., $d=0.701$ ), the expert will, sometimes, not be able to give a definite answer, he will be uncertain about it. This uncertainty can be, in its turn, described by a number from the interval $[0,1]$. It is, therefore, natural to represent our degree of certainty in a statement $A$ not by a single (crisp) number $d(A) \in[0,1]$ (as in the [0,1]-based description), but rather by a function $\mu_{\mathbf{d}(A)}$ which assigns, to each possible real number $d \in[0,1]$, a degree $\mu_{\mathbf{d}(A)}(d)$ with which this number $d$ can be the (desired) degree of certainty of $A$. This is called a second-order description of uncertainty. General second order descriptions have been successfully used in many practical applications ranging from control (including robotic control) to medicine to military decision-making; see, e.g., [32,33,37,44].

From second order to general higher order approach. In second-order description, to describe a degree with which a given number $d \in[0,1]$ can be a degree of certainty of a statement $A$, we use a real number $\mu_{\mathbf{d}(A)}(d)$. As we have already mentioned, it is difficult to describe our degree of certainty by a single number. Therefore, to make this description even more realistic, we can represent each degree of certainty $d(P(x))$ not by a (more traditional) $[0,1]$-based description, but by a second order description. As a result, we get the third order description.

Similarly, to make our description even more realistic, we can use the third order descriptions to describe degrees of certainty; then, we get fourth order uncertainty, etc.

Towards combining the granularity and higher-order approaches. Both granularity and second order approaches aim at the same goal: to make a computer representation of expert knowledge closer to the actual human reasoning. The fact that both approaches resulted in successful practical applications indicate that these two approaches capture important aspects of 
human reasoning. The fact that each approach is successful in some applications where the other approach did not succeed so well shows that these approaches capture different aspects of human reasoning. It is therefore desirable to combine these two approaches.

This idea of combining the two approaches is very natural, and it has been actually tried. However, so far, it has led to few successful practical applications. It is therefore desirable to find an optimal way of combining these approaches.

What we are planning to do. This chapter presents the results of the authors' preliminary research in this direction. Before deciding what is the best way to combine these two approaches, we should decide which versions of these approaches are the best to use (and to combine). We start with granularity. In Section 2, we show, on some reasonable examples, what is the optimal granularity. In Section 3, we handle the problem of selecting operations that are in optimal agreement with the granularity. In Section 4 , we use the above-described "common origin" of granularity and higherorder logics to find out which of the higher-order approaches is optimal. Finally, in Section 5, we provide preliminary results about operations which are consistent both with granularity and higher-order logics.

This chapter is largely an overview of the results published by the authors in several conference papers $[18,21,22,40]$. In each of these results, we:

- provide a formalization of the corresponding problem;

- provide a solution to this problem - if such a general solution is possible at all - we will prove that for some of these problems, a general solution is not possible; and

- illustrate the problem and the solution on one or several examples.

Most formalizations, solutions, and proofs from this chapter are the authors'; the missing proofs can be found in the above-cited conference proceedings.

A few words about our choice of examples. Our main goal is to provide an adequate description of expert reasoning. Therefore, to gauge how well we achieved this goal, we must show, on examples, that our approach is more adequate in describing expert reasoning than the traditional [0,1]-based fuzzy approach. To make this comparison convincing, we must consider cases when there is no disagreement on how an expert reached her conclusion. Such cases are extremely rare in real-life expert reasoning: for example, it is very difficult for a lay person to understand why an emergency room medical doctor makes a decision that may save or ruin the life of a patient. It is difficult to understand because if such decisions were easy, we would not need experts to make these decisions. Since it is difficult to use real-life expert decisions to compare the new methods with the more traditional ones, we therefore use simplified "toy" examples for this comparison, examples where - unlike most real-life situations - reasoning is easy to understand. 


\section{Optimal Granularity: Case Study}

Granularity: case study. In accordance with our plan, let us start with describing an example in which we can determine the optimal granularity. This example relates to crude estimates of quantities.

People often need to make crude estimates of a quantity, e.g., estimating the size of a crowd or someone's salary. When people make these crude estimates, they usually feel reasonably comfortable choosing between alternatives which differ by a half order of magnitude (HOM). For example, a person can reasonably estimate whether the size of a crowd was closer to 100 , or to 300 , or to 1000 . If we ask for an estimate on a more refined scale, e.g., 300 or 350 , people will generally be unable to make it. If we ask for an estimate on a coarser scale, e.g., 100 or 1000, people may be able to answer, but they will feel their answer is uninformative.

A particularly striking case of the utility of HOMs is presented by coinage and currency. Most countries have, in addition to denominations for the powers of ten, one or two coins or bills between every two powers of ten. Thus, in the United States, in addition to coins or bills for $\$ .01, \$ .10, \$ 1.00, \$ 10.00$, and $\$ 100.00$, there are also coins or bills in common use for $\$ .05, \$ .25, \$ 5.00$, $\$ 20,00$, and $\$ 50.00$. These latter provide rough HOM measures for monetary amounts.

It is natural that people should categorize the sizes of physical objects in terms of how they must interact with them. When two objects are roughly of the same size, we manipulate them or navigate about them in roughly the same way. But when one object is about three times larger in linear dimension than another, it must be handled in a different manner. Thus, an orange can be held in one hand, whereas a basketball is more easily held with two, A carton is held in our arms rather than our hands, and carrying a table often requires a second person. For further arguments along these lines, see [17].

These observations lead naturally to the following question: If we are to have a rough logarithmic classification scheme for quantities, what is the optimal granularity for commonsense estimates?

There are three requirements we would like the classification scheme to have.

- The categories should be small enough that the types of our interactions with objects are predictable from their category; that HOMs accomplish this is argued above and in [17].

- The categories should be large enough that ordinary variation among objects in a class do not usually cross category boundaries and that aggregation operations have reasonably predictable results; we show that HOMs satisfy these requirements.

Thus we describe two different models for commonsense estimation and show that in both models the optimal granularity is in good accordance with obser- 
vations about the utility of HOMs. We thus provide a theoretical explanation for the importance of half orders of magnitude in commonsense reasoning.

Main idea behind Gaussian model. We are interested in the situation where we estimate a quantity which can only take non-negative values. To estimate the values of this quantity, we select a sequence of positive numbers $\ldots<e_{0}<e_{1}<e_{2}<\ldots$ (e.g., $1,3,10$, etc.), and every actual value $x$ of the estimated quantity is then estimated by one of these numbers. Each estimate is approximate: when the estimate is equal to $e_{i}$, the actual value $x$ of the estimated quantity may differ from $e_{i}$; in other words, there may be an estimation error $\Delta x=e_{i}-x \neq 0$.

What is the probability distribution of this estimation error? This error is caused by many different factors. It is known that under certain reasonable conditions, an error caused by many different factors is distributed according to Gaussian (normal) distribution (see, e.g., [53]; this fact - called central limit theorem - is one of the reasons for the widespread use of Gaussian distribution in science and engineering applications). It is therefore reasonable to assume that $\Delta x$ is normally distributed.

It is known that a normal distribution is uniquely determined by its two parameters: its average $a$ and its standard deviation $\sigma$. Let us denote the average of the error $\Delta x$ by $\Delta e_{i}$, and its standard deviation by $\sigma_{i}$. Thus, when the estimate is $e_{i}$, the actual value $x=e_{i}-\Delta x$ is distributed according to Gaussian distribution, with an average $e_{i}-\Delta e_{i}$ (which we will denote by $\widetilde{e}_{i}$ ), and the standard deviation $\sigma_{i}$.

For a Gaussian distribution with given $a$ and $\sigma$, the probability density is everywhere positive, so theoretically, we can have values which are as far away from the average $a$ as possible. In practice, however, the probabilities of large deviations from $a$ are so small that the possibility of such deviations can be safely neglected. For example, it is known that the probability of having the value outside the "three sigma" interval $[a-3 \sigma, a+3 \sigma]$ is $\approx 0.1 \%$ and therefore, in most engineering applications, it is assumed that values outside this interval are impossible.

There are some applications where we cannot make this assumption. For example, in designing computer chips, when we have millions of elements on the chip, allowing $0.1 \%$ of these elements to malfunction would mean that at any given time, thousands of elements malfunction and thus, the chip would malfunction as well. For such critical applications, we want the probability of deviation to be much smaller than $0.1 \%$, e.g., $\leq 10^{-8}$. Such small probabilities (which practically exclude any possibility of an error) can be guaranteed if we use a "six sigma" interval $[a-6 \sigma, a+6 \sigma]$. For this interval, the probability for a normally distributed variable to be outside it is indeed $\approx 10^{-8}$.

Within this Gaussian model, what is the optimal granularity? 
Optimal granularity: informal explanation. In accordance with the above idea, for each $e_{i}$, if the actual value $x$ is within the "three sigma" range $I_{i}=\left[\widetilde{e}_{i}-3 \sigma_{i}, \widetilde{e}_{i}+3 \sigma_{i}\right]$, then it is reasonable to take $e_{i}$ as the corresponding estimate.

We want a granulation which would cover all possible values, so each positive real number must be covered by one of these intervals. In other words, we want the union of all these intervals to coincide with the set of all positive real numbers.

We also want to makes sure that all values that we are covering are indeed non-negative, i.e., that for every $i$, even the extended "six sigma" interval

$$
\left[\widetilde{e}_{i}-3 \sigma_{i}, \widetilde{e}_{i}+3 \sigma_{i}\right]
$$

only contains non-negative values.

Finally, since one of the main purposes of granularity is to decrease the number of "labels" that we use to describe different quantities, we want to consider optimal (minimal) sets of intervals. Formally, we can interpret "minimal" in the sense that whichever finite subset we pick, we cannot enlarge their overall coverage by modifying one or several of these intervals. Let us formalize these ideas.

In the following definitions, we will use the fact that an arbitrary interval $\left[a^{-}, a^{+}\right]$can be represented in the Gaussian-type form $[a-3 \sigma, a+3 \sigma]$ : it is sufficient to take $a=\left(a^{-}+a^{+}\right) / 2$ and $\sigma=\left(a^{+}-a^{-}\right) / 6$.

\section{Definition 2.1.}

- We say that an interval $I=[a-3 \sigma, a+3 \sigma]$ is reliably non-negative if every real number from the interval $[a-6 \sigma, a+6 \sigma]$ is non-negative.

- A set $\left\{I_{i}\right\}, i=1,2, \ldots$, of reliably non-negative intervals $I_{i}$ is called a granulation if every positive real number belongs to one of the intervals $I_{i}$.

- We say that a granulation can be improved if, for some finite set $\left\{i_{1}, \ldots, i_{k}\right\}$, we can replace intervals $I_{i_{j}}$ with some other intervals $I_{i_{j}}^{\prime}$ for which

$$
\bigcup_{j=1}^{k} I_{i_{j}} \subset \bigcup_{j=1}^{k} I_{i_{j}}^{\prime} \quad \bigcup_{j=1}^{k} I_{i_{j}} \neq \bigcup_{j=1}^{k} I_{i_{j}}^{\prime},
$$

and still get a granulation.

- A granulation is called optimal if it cannot be improved.

Theorem 2.1. (Hobbs and Kreinovich [18]) In an optimal granulation, $I_{i}=$ $\left[a_{i}, a_{i+1}\right]$, where $a_{i+1}=3 a_{i}$.

So, half-orders of magnitude are indeed optimal.

Uniform model: motivations. In the Gaussian model, we started with a $3 \sigma$ bound, and we ended up with a sequence of granules $\left[a_{i}, a_{i+1}\right]$ in which the boundary points $a_{i}$ form an arithmetic progression: $a_{i+1}=q \cdot a_{i}$ and $a_{i}=a_{0} \cdot q^{i}$, with $q=3$. We could start with a bound of $2.5 \sigma$, then we would 
have got a geometric progression with a different $q$. Which value of $q$ is indeed optimal?

To find out, let us take into consideration the fact that a granulation is not just for storing values, it is also for processing these values. Of course, when we replace the actual value by the granule to which it belongs, we lose some information. The idea is to choose the $q$ for which this loss is the smallest.

To estimate the loss, we will consider the simplest data processing operation possible: addition. If we know the exact values of two quantities $A$ and $B$, then we can compute the exact value of their sum $A+B$. In the granulated case, we do not know the exact values of $A$ and $B$, we only know the granules to which $A$ and $B$ belong, and we want to find out to which of the granules the sum belongs. For example, in the above half-order granulation, we know that the first room has about 10 books, the second about 30, and we want to express the total number of books in the two rooms in similar terms.

The trouble with this problem is that the sum may belong to two different granules. Let us take an example in which we use granules [1,3], [3, 9], $[9,27]$, etc. Let us assume that all we know about the first quantity $A$ is that $A \in$ $[1,3]$, and all we know about the second quantity $B$ is that $B \in[3,9]$. In this case, the smallest possible values of $A+B$ is $1+3=4$, and the largest possible value of $A+B$ is $3+9=12$. In general, the sum $A+B$ can thus take any value from the interval $[4,12]$. So, it could happen that the sum is in the granule $[3,9]$, but it could also happen that the sum is in the granule $[9,27]$.

If we want the granulation to be useful, we must assign a certain granule to the sum $A+B$. Since in reality, the value $A+B$ may belong to two different granules, no matter which of the two granules we assign, there is always a probability that this assignment is erroneous. We would like to select $q$ for which this error probability is the smallest possible.

In order to formulate this question in precise terms, we must describe the corresponding probabilities. A natural way to describe them is as follows: If all we know about $A$ is that $A$ belongs to a granule $\mathbf{a}_{i}=\left[a_{i}, a_{i+1}\right]$, then it is reasonable to consider all the values from this granule to be equally probable, i.e., to assume that we have a uniform distribution on the interval $\mathbf{a}_{i}=\left[a_{i}, a_{i+1}\right]$. Similarly, If all we know about $B$ is that $B$ belongs to a granule $\mathbf{a}_{j}=\left[a_{j}, a_{j+1}\right]$, then it is reasonable to consider all the values from this granule to be equally probable, i.e., to assume that we have a uniform distribution on the interval $\mathbf{a}_{j}=\left[a_{j}, a_{j+1}\right]$. Since we have no information about the possible dependence between $A$ and $B$, it is natural to assume that $A$ and $B$ are independent random variables. We are now ready for the formal definitions.

Let $a_{0}>0$ and $q \geq 2$ be real numbers, and let $a_{k} \stackrel{\text { def }}{=} a_{0} \cdot q^{k}$ and $\mathbf{a}_{i} \stackrel{\text { def }}{=}$ $\left[a_{i}, a_{i+1}\right]$. 
Definition 2.2. For every three integers $i, j$, and $k$, we can define

$$
P\left(\mathbf{a}_{i}+\mathbf{a}_{j} \in \mathbf{a}_{k}\right)
$$

as the probability that $A_{i}+A_{j} \in \mathbf{a}_{k}$, where $A_{i}$ is uniformly distributed on the interval $\mathbf{a}_{i}, A_{j}$ is uniformly distributed on the interval $\mathbf{a}_{j}$, and $A_{i}$ and $A_{j}$ are independent.

If, as a result of adding $\mathbf{a}_{i}$ and $\mathbf{a}_{j}$, we select the granule $\mathbf{a}_{k}$, then the probability that this assignment is erroneous (i.e., that the actual value of $A_{i}+A_{j}$ is not in $\left.\mathbf{a}_{k}\right)$ is equal to $1-P\left(\mathbf{a}_{i}+\mathbf{a}_{j} \in \mathbf{a}_{k}\right)$. For every $i$ and $j$, we want to minimize this error, so we select the value $k$ for which this error probability is the smallest:

Definition 2.3. For every two integers $i$ and $j$, we define the sum $\mathbf{a}_{i}+\mathbf{a}_{j}$ of granules $\mathbf{a}_{i}$ and $\mathbf{a}_{j}$ as a granule $\mathbf{a}_{k}$ for which the error probability

$$
1-P\left(\mathbf{a}_{i}+\mathbf{a}_{j} \in \mathbf{a}_{k}\right)
$$

is the smallest possible. The error probability $E_{i j}$ related to this addition is then defined as this smallest probability, i.e., as

$$
E_{i j} \stackrel{\text { def }}{=} \min _{k}\left(1-P\left(\mathbf{a}_{i}+\mathbf{a}_{j} \in \mathbf{a}_{k}\right)\right)
$$

Theorem 2.2. (Hobbs and Kreinovich [18]) When $q \geq \sqrt{2}+1(\approx 2.41)$, then

$$
\mathbf{a}_{i}+\mathbf{a}_{i}=\mathbf{a}_{i+1}
$$

and

$$
\mathbf{a}_{i}+\mathbf{a}_{j}=\mathbf{a}_{\max (i, j)} \text { for } i \neq j .
$$

When $2 \leq q<\sqrt{2}+1$, then $\mathbf{a}_{i}+\mathbf{a}_{i}=\mathbf{a}_{i+1}$,

$$
\mathbf{a}_{i}+\mathbf{a}_{i+1}=\mathbf{a}_{i+1}+\mathbf{a}_{i}=\mathbf{a}_{i+2}
$$

and

$$
\mathbf{a}_{i}+\mathbf{a}_{j}=\mathbf{a}_{\max (i, j)} \text { for }|i-j| \geq 2 \text {. }
$$

It is worth mentioning that for every $q$, thus defined addition of granules is commutative but not associative. Indeed, for $q \geq \sqrt{2}+1$, we have:

$$
\left(\mathbf{a}_{0}+\mathbf{a}_{0}\right)+\mathbf{a}_{1}=\mathbf{a}_{1}+\mathbf{a}_{1}=\mathbf{a}_{2},
$$

while

$$
\mathbf{a}_{0}+\left(\mathbf{a}_{0}+\mathbf{a}_{1}\right)=\mathbf{a}_{0}+\mathbf{a}_{1}=\mathbf{a}_{1} \neq \mathbf{a}_{2}
$$

For $q<\sqrt{2}+1$, we have: 


$$
\left(\mathbf{a}_{0}+\mathbf{a}_{0}\right)+\mathbf{a}_{2}=\mathbf{a}_{1}+\mathbf{a}_{2}=\mathbf{a}_{3},
$$

while

$$
\mathbf{a}_{0}+\left(\mathbf{a}_{0}+\mathbf{a}_{2}\right)=\mathbf{a}_{0}+\mathbf{a}_{2}=\mathbf{a}_{2} \neq \mathbf{a}_{3} .
$$

Which $q$ is the best? As a measure of quality of a given granulation, it is natural to take the worst-case error probability, i.e., the error probability corresponding to the worst-case pair $(i, j)$ (i.e., to the pair with the largest $E_{i j}$ ):

Definition 2.4. By an error probability of a granulation, we mean the value $E(q) \stackrel{\text { def }}{=} \max _{i, j} E_{i j}$. The granulation with the smallest possible error probability is called optimal.

Theorem 2.3. (Hobbs and Kreinovich [18]) The granulation is optimal when

$$
q^{3}-5 q^{2}+4 q+1=0
$$

(i.e., when $q \approx 3.9$ ).

Conclusion. When people make crude estimates, they feel comfortable choosing between alternatives which differ by a half-order of magnitude (e.g., were there 100,300 , or 1,000 people in the crowd), and less comfortable making a choice on a more detailed scale (like 100 or 110 or 120) or on a coarser scale (like 100 or 1,000). We have shown that for two natural models of choosing granularity in commonsense estimates, in the optimal granularity, the next estimate is 3-4 times larger than the previous one. Thus, we have explained the commonsense HOM granularity.

\section{Selecting Operations that Are in Optimal Agreement with Granularity}

Intuitive property of commonsense arithmetic. To explain the problem that we try to solve in this section, let us start with a joke that nicely illustrates the notion of granularity and the difficulty of handling granular data.

A museum guide tells the visitors that a dinosaur that they are looking at is 14,000,005 years old. An impressed visitor asks how scientists can be so accurate in their predictions. "I don't know how they do it, - explains the guide - but 5 years ago, when I started working here, I was told that this dinosaur is 14,000,000 years old, so now it must be 5 years older".

This is clearly a joke, because from the common sense viewpoint, a dinosaur which was approximately 14,000,000 years old 5 years ago is still $14,000,000$ years old. In more precise terms, if we add 5 to a "fuzzy" number "approximately 14,000,000", we should get the answer "approximately $14,000,000 "$. 
Similarly, if a person weighs, say, approximately $100 \mathrm{~kg}$, and he gains 1 $\mathrm{kg}$, he still weighs approximately $100 \mathrm{~kg}$. So, if we add 1 to a "fuzzy" number "approximately 100", we should get the answer "approximately 100".

In general, if $a$ is much larger than $b(a \gg b)$, and we add $b$ to "approximately $a$ ", we should get "approximately $a$ ". It is therefore natural to expect formal systems which formalize commonsense reasoning to have this property.

Fuzzy arithmetic: a natural formalization of commonsense arithmetic. A natural way of dealing with approximately known values (such as "approximately a") is fuzzy arithmetic. In fuzzy arithmetic, each such value is represented by a membership function $\mu(x)$ describing, for each real number $x$, to what extent $x$ matches the description (see, e.g., $[19,45]$ ).

For example, if the value that we want to formalize is "approximately $a$ " (for some given real number $a$ ), then the value $x=a$ matches the described property perfectly well $(\mu(a)=1)$, while the more distant the value $x$ from $a$, the smaller the degree of matching. In other words, a natural way to represent a property "approximately $a$ " is to have a membership function $\mu(x)$ which:

- attains its maximum value 1 for $x=a$,

- increases for $x<a$, and

- decreases for $x>a$.

In practical applications, researchers have used membership functions $\mu(x)$ of different shape to represent the property "approximately $a$ ": Gaussian, piece-wise linear, etc.; all these shapes have a clear maximum at $x=a$.

Vice versa, if we have a membership function $\mu(x)$ which:

- has a clear maximum at some point $x=a$,

- is increasing for $x<a$, and

- is decreasing for $x>a$,

it is natural to interpret this function as describing a property "approximately $a "$.

When several numbers $A, B$, etc., are described by membership functions, we can use the extension principle to describe the result of applying an arithmetic operation to these numbers. For example, if a number $A$ is described by a membership function $\mu_{A}(x)$, and the number $B$ is described by a membership function $\mu_{B}(x)$, then their sum $C=A+B$ is described by the following membership function:

$$
\mu_{C}(x)=\max _{y, z: y+z=x} \min \left(\mu_{A}(y), \mu_{B}(z)\right) .
$$

We can also have a more general formula, if we use an arbitrary t-norm instead of the minimum.

Whether we use min or a more general t-norm, in the simple case when the number $B$ is crisp $(B=b)$, the resulting membership function is equal to 
$\mu_{C}(x)=\mu_{A}(x-b)$; in other words, it has the same shape as the membership function for $A$ - but it is shifted by $b$.

Problem: traditional fuzzy arithmetic does not have the desired property. In many practical applications, the traditional fuzzy arithmetic works well. Unfortunately, the traditional fuzzy arithmetic does not satisfy the desired intuitive property.

Indeed, let $A$ mean "approximately a" (e.g., "approximately 100"). Then, the corresponding membership function $\mu_{A}(x)$ has a maximum at $x=a$, is increasing for $x<a$ and decreasing for $x>a$. When we add, to $A$, a crisp number $B=b$ (e.g., 1 ), we get a shifted membership function which has a maximum at $x=a+b$, is increasing for $x<a+b$ and decreasing for $x>a+b$. In accordance with the above interpretation, we thus interpret the sum $A+B$ as "approximately $a+b$ ". Thus, the sum " $\approx 100 "+1$ is equal not to $\approx 100$ as we would intuitively expect, but to $\approx 101$.

Trapezoidal membership functions: can they help? At first glance, we may think that the above problem of fuzzy arithmetic comes from the fact that we considered fuzzy sets $A(x)$ that have a maximum only at a single point $x=a$. Maybe, to represent notions like the age of dinosaurs, we should consider fuzzy sets with interval "core", i.e., fuzzy sets $A(x)$ which attain the maximum at the entire interval. The most widely used examples of such fuzzy sets are trapezoidal sets. Sets with interval core are clearly more suitable, but do they solve the problem?

Let us consider such sets. To describe the property "approximately $a$ ", we usually consider "symmetric" fuzzy sets, i.e., fuzzy sets $\mu(x)$ in which the value $\mu(x)$ depend only on the distance between $x$ and $a$, i.e., in which, for each distance $d>0$, the degrees $\mu(a-d)$ and $\mu(a+d)$ are equal. For such sets, the interval core is centered around $a$, i.e., has the form $[a-\Delta, a+\Delta]$ for some value $\Delta>0$. The property "approximately $a$ " is thus represented by a membership function $\mu(x)$ which:

- attains its maximum value 1 for $x$ from the interval $[a-\Delta, a+\Delta]$;

- increase for $x<a-\Delta$, and

- decreases for $x>a+\Delta$.

Vice versa, if we have a membership function $\mu(x)$ which satisfies these three properties for some value $a$, then it is natural to interpret this function as describing a property "approximately $a$ ".

If $A$ mean "approximately a" (e.g., "approximately 100"), then, the corresponding membership function $\mu_{A}(x)$ has a maximum for $x \in[a-\Delta, a+\Delta]$, is increasing for $x<a-\Delta$ and decreasing for $x>a+\Delta$. When we add, to $A$, a crisp number $B=b$ (e.g., 1), we get a shifted membership function which has a maximum $x \in[\widetilde{a}-\Delta, \widetilde{a}+\Delta]$ (where $\widetilde{a} \stackrel{\text { def }}{=} a+b$ ), is increasing for $x<\widetilde{a}-\Delta$ and decreasing for $x>\widetilde{a}+\Delta$. In accordance with the above interpretation, we thus interpret the sum $A+B$ as "approximately $\widetilde{a}$ ", i.e., 
"approximately $a+b "$. Thus, the sum " $\approx 100 "+1$ is equal not to $\approx 100$ as we would intuitively expect, but to $\approx 101$.

Changing the shape of the membership function does not help. We must therefore modify the operations of fuzzy arithmetic. How can we modify fuzzy arithmetic to make sure that the desired property is satisfied, and the sum of " $\approx 100 "$ and 1 is equal to $\approx 100$ ?

Main idea. When we only know a (crisp of fuzzy) interval of possible values of a certain quantity (or a more general set of possible values), it is desirable to characterize this interval by supplying the user with the "simplest" element from this interval, and by characterizing how far away from this value we can get. For example, if, for some unknown physical quantity $x$, measurements result in the interval $[1.95,2.1]$ of possible values, then, most probably, the physicist will publish this result as $y \approx 2$. Similarly, a natural representation of the measurement result $x \in[3.141592,3.141593]$ is $x \approx \pi$.

So, intuitively, if we know the membership functions for $A$ and for $B$, we should:

- compute the membership function $\mu_{C}(x)$ for $C=A+B$;

- find the interval of possible values of $C$ (e.g., as all the values for which $\mu_{C}(x) \geq d_{0}$ for some value $\left.d_{0}\right)$;

- pick the simplest value $c$ on this interval, and then

- return "approximately $c$ " as the result of adding $A$ and $B$.

In particular, when $A$ is "approximately 14,000,000" - meaning that the interval of possible values is probably $[13,500,000 ; 14,500,000]-$ and $B$ is a crisp value 5 , then for $A+B$, the interval of possible values is $[13,500,005$; $14,500,005]$. On this interval, $14,000,000$ is probably still the simplest value, so we conclude that the sum of "approximately 14,000,000" and 5 is - as we expected - equal to "approximately 14,000,000".

Similarly, in this new definition, if we add $1 \mathrm{~kg}$ to a weight of approximately $100 \mathrm{~kg}$, we still get approximately $100 \mathrm{~kg}$ as the result.

How to formalize this definition? In order to formalize the above definition, we must formalize what "simplest" means. Intuitively, the simpler the description of a real number, the simpler this number. Thus, to define relative complexity of different real numbers, we fix some logical theory $T$ in which we will describe real numbers.

We will consider languages in which the list of sorts $\mathcal{S}$ contains two symbols: "integer" and "real", and which contain standard arithmetic predicates and function symbols such as $0,1,+,-, \cdot, /,=,<, \leq$, both for integers and for reals. We will assume that this theory contains both the standard first order theory of integers (Peano arithmetic $[3,11,48]$ ) and a standard first order theory of real numbers $[5,10,49,51]$. One of the possibilities is to consider, as the theory $T$, axiomatic set theory (e.g., ZF), together with explicit definitions of integers, real numbers, and standard operations and predicates in terms of set theory. 
Once a theory $T$ is fixed, we can define a complexity $D(x)$ of a real number $x$ as the shortest length of a formula $F(y)$ in the language $L$ which defines this particular number $x$, i.e., which is true for $y=x$ and false for $y \neq x$.

To clarify this definition, let us give examples of formulas which define different real numbers:

- A formula $(y \cdot y=1+1) \& y \geq 0$ is true if and only if $y=\sqrt{2}$; thus, this formula defines the number $\sqrt{2}$.

- Similarly, a formula $\forall x(x \cdot y=x+x+x)$ defines a real number 3 .

- If the language of the theory $T$ contains the sine function sin, and if the corresponding theory contains the standard definition of the sine function, then the formula $\sin (y)=0 \& 3 \leq y \leq 4$ defines a real number $\pi$.

Comment 1. This definition is similar to the so-called Kolmogorov complexity $C(x)$ (invented independently by Chaitin, Kolmogorov, and Solomonoff), which is defined as the smallest length of the program that computes $x$ (for a current survey of Kolmogorov complexity, see, e.g., [27]). In our case, however, we do not care that much about how to compute: computing 3.141592 may be easier than computing $\pi$; we are more interested in how easy it is to describe $x$. Due to this difference, we cannot simply use the original Kolmogorov's definition: we have to modify it.

Comment 2. It is worth mentioning that not all real numbers are definable: indeed, there are only countably many formulas, so there can be no more than countably many definable real numbers, while the total cardinality of the set of all real numbers is known to be larger $\left(\aleph_{1}>\aleph_{0}\right)$.

This new definition solves the above problem, but - in full accordance with the saying "there is no free lunch" - it comes with drawbacks. We will see that these drawbacks do not mean that our solution is bad, they seem to be implied (surprisingly) by the very properties that we try to retain.

First drawback: addition is no longer always associative. This drawback is the easiest to describe and to explain. Both standard arithmetic and traditional fuzzy arithmetic are associative: if we add several numbers $A_{1}+\ldots+A_{n}$, the resulting sum does not depend on the order in which we add them; in particular,

$$
\begin{gathered}
\left(\ldots\left(\left(A_{1}+A_{2}\right)+A_{3}\right)+\ldots\right)+A_{n}= \\
A_{1}+\left(A_{2}+\left(A_{3}+\left(\ldots+A_{n}\right) \ldots\right)\right) .
\end{gathered}
$$

Let us show that for the newly defined addition, this formula is no longer always true.

Indeed, suppose now that we want to formalize the idea that, say " $~ 100$ " +1 is equal to $\approx 100$ (this is just an example, but any other example can be used to illustrate non-associativity). Let us take $n=101$, "approximately $100 "$ as $A_{1}$, and $A_{2}=\ldots=A_{n}=1$ (crisp numbers). In terms of the newly defined numbers $A_{i}$, the desired property takes the form $A_{1}+A_{2}=A_{1}$ 
(similarly, $A_{1}+A_{3}=A_{1}$, etc.). Thus, $A_{1}+A_{2}=A_{1}$, hence $\left(A_{1}+A_{2}\right)+A_{3}=$ $A_{1}+A_{3}=A_{1}$, etc., and hence the left-hand side of the formula (16) is equal to "approximately 100":

$$
\left(\ldots\left(\left(A_{1}+A_{2}\right)+A_{3}\right)+\ldots\right)+A_{n}=A_{1} .
$$

On the other hand, since $A_{2}, \ldots, A_{n}$ are crisp numbers (equal to 1 each), their sum $A_{2}+\left(A_{3}+\left(\ldots+A_{n}\right) \ldots\right)$ is simply a crisp number $1+\ldots+1=100$. Thus, the right-hand side of the formula (16) is equal to

$$
\text { "approximately 100" + } 100
$$

which, intuitively, should be rather "approximately 200" than "approximately 100 ". Thus, the left-hand side of (16) is clearly different from its right-hand side. Hence, the newly defined addition is not associative.

Second drawback: addition is no longer always easily computable. Traditional fuzzy arithmetic - defined by the extension principle - provides an explicit formula for computing the sum $C=A+B$ of two fuzzy numbers $A$ and $B$. So, we can still find the interval of possible values for $C$. Unfortunately, as we will now show, the next step - finding the simplest possible real number on this interval - is no longer easily computable.

Theorem 3.1. (Kreinovich, Nguyen, and Pedrycz [22]) No algorithm is possible that, given an interval with definable endpoints, would return the simplest real number from this interval.

A similar result holds for computable real numbers. A similar result holds if we restrict ourselves to computable real numbers, i.e., real numbers that can be computed with an arbitrary accuracy (see, e.g., $[4,6,7,9]$ ). To be more precise, a real number $x$ is called computable if there exists an algorithm (program) that transforms an arbitrary integer $k$ into a rational number $x_{k}$ that is $2^{-k}$-close to $x$. It is said that this algorithm computes the real number $x$.

Every computable real number is uniquely determined by the corresponding algorithm and is, therefore, definable.

Theorem 3.2. (Kreinovich, Nguyen, and Pedrycz [22]) No algorithm is possible that, given an interval with computable endpoints, returns the simplest computable real number from this interval.

Conclusion. From the commonsense viewpoint, if 5 years ago, a dinosaur was approximately $14,000,000$ years old, it is still approximately 14,000,000 years years old. Unfortunately, when we formalize the notion "approximately $14,000,000$ " in traditional fuzzy arithmetic, we do not get this property. In this section, we have described a natural modification of fuzzy arithmetic which does have this property. This modification is closer to commonsense reasoning, but this closeness comes at a cost: addition is no longer always associative and no longer always easily computable. 


\section{Optimal Selection of Higher-Order Approach}

Third order descriptions are not used: why? Theoretically, we can define third, fourth order, etc., descriptions, but in practical applications, only second order descriptions were used so far (see, e.g., $[32,33,37,44]$ ). Based on this empirical fact, it is natural to conclude that third and higher order descriptions are not really necessary. In this section, we will use the relation with granularity to show that this empirical conclusion can be theoretically justified.

First step in describing uncertainty: set of uncertainty-describing words. Let us first describe the problem formally. An expert uses words from a natural language to describe his degrees of certainty. In every language, there are only finitely many words, so we have a finite set of words ("granules") that need to be interpreted. We will denote this set of words by $W$.

Second step: a fuzzy property described by a word-valued "membership function". If we have any property $P$ on a universe of discourse $U$, an expert can describe, for each element $x \in U$, his degree of certainty $d(x) \in W$ that the element $x$ has the property $P$.

Traditional fuzzy logic as a first approximation: numbers assigned to words describing uncertainty. Our ultimate goal is to provide a computer representation for each word $w \in W$. In the traditional [0,1]-based description, this computer representation assigns, to every word, a real number from the interval $[0,1]$; in general, we may have some other computer representations (examples will be given later). Let us denote the set of all possible computer representations by $S$.

In the first approximation, i.e., in the first order description, we represent each word $w \in W$, which describes a degree of uncertainty, by an element $s \in S$ (e.g., by a real number from the interval $[0,1]$ ). In this section, we will denote this first-approximation computer representation of a word $w$ by $s=\|w\|$.

If the set $S$ is too small, then it may not contain enough elements to distinguish between different expert's degree of belief: this was exactly the problem with classical $\{0,1\}$-based description, in which we only have two possible computer representations - "true" and "false" - that are not enough to adequately describe the different degrees of certainty. We will therefore assume that the set $S$ is rich enough to represent different degrees of certainty.

In particular, the set $[0,1]$ contains infinitely many points, so it should be sufficient; even if we only consider computer-representable real numbers, there are still much more of them (millions and billions) than words in a language (which is usually in hundreds of thousands at most), so we can safely make this "richness" assumption. In mathematical terms, it means that two different degrees of belief are represented by different computer terms, i.e., that if $w_{1} \neq w_{2}$, then $\left\|w_{1}\right\| \neq\left\|w_{2}\right\|$. 
First approximation is not absolutely adequate. The problem with the first-order representation is that the relation between words $w \in W$ and computer representation $s \in S$ is, in reality, also imprecise. Typically, when we have a word $w \in W$, we cannot pick a single corresponding representative $s \in S$; instead, we may have several possible representatives, with different degrees of adequacy.

Actual description of expert uncertainty: word-valued degree to which a word describes uncertainty. In other words, instead of a single value $s=\|w\|$ assigned to a word $w$, we have several values $s \in S$, each with its own degree of adequacy; this degree of adequacy can also be described by an expert, who uses an appropriate word $w \in W$ from the natural language.

In other words, for every word $w \in W$ and for ever representation $s \in S$, we have a degree $w^{\prime} \in W$ describing to what extent $s$ is adequate in representing $w$. Let us represent this degree of adequacy by $a(w, s)$; the symbol $a$ represents a function $a: W \times S \rightarrow W$, i.e., a function that maps every pair $(w, s)$ into a new word $a(w, s)$.

Second-order description of uncertainty as a second approximation to actual uncertainty. So, the meaning of a word $w \in W$ is represented by a function $a$ which assigns, to every element $s \in S$, a degree of adequacy $a(w, s) \in W$. We want to represent this degree of adequacy in a computer; therefore, instead of using the word $a(w, s)$ itself, we will use the computer representation $\|a(w, s)\|$ of this word. Hence, we get a second-order representation, in which a degree of certainty corresponding to a word $w \in W$ is represented not by a single element $\|w\| \in S$, but by a function $\mu_{w}: S \rightarrow S$, a function which is defined as $\mu_{w}(s)=\|a(w, s)\|$.

Second-order description is not $100 \%$ adequate either; third-, fourth-order descriptions, etc. The second-order representation is also not absolutely adequate, because, to represent the degree $a(w, s)$, we used a single number $\|a(w, s)\|$. To get a more adequate representation, instead of this single value, we can use, for each element $s^{\prime} \in S$, a degree of adequacy with which the element $s^{\prime}$ represents the word $a(w, s)$. This degree of adequacy is also a word $a\left(a(w, s), s^{\prime}\right)$, so we can represent it by an appropriate element $\left\|a\left(a(w, s), s^{\prime}\right)\right\|$. Thus, we get a third-order representation, in which to every element $s$, we assign a second-order representation. To get an even more adequate representation, we can use fourth- and higher order representations.

Let us express this scheme formally.

\section{Definition 4.1.}

- Let $W$ be a finite set; element of this set will be called words.

- Let $U$ be a set called a universe of discourse.

- By a fuzzy property $P$, we mean a mapping which maps each element $x \in U$ into a word $P(x) \in W$; we say that this word described the degree of certainty that $x$ satisfies the property $P$. 
Definition 4.2. By a first-approximation uncertainty representation, we mean a pair $\langle S,\|\cdot\|\rangle$, where:

- $S$ is a set; elements of this set will be called computer representations; and

- $\|$.$\| is a function from W$ to $S$; we say that an element $\|w\| \in S$ represents the word $w$.

We say that an uncertainty representation is sufficiently rich if for every two words $w_{1}, w_{2} \in W, w_{1} \neq w_{2}$ implies $\left\|w_{1}\right\| \neq\left\|w_{2}\right\|$.

Definition 4.3. Let $W$ be a set of words, and let $S$ be a set of computer representations. By an adequacy function, we mean a function $a: W \times S \rightarrow W$; for each word $w \in W$, and for each representation $s \in S$, we say that $a(w, s)$ describes the degree to which the element $s$ adequately describes the word $w$.

Definition 4.4. Let $U$ be a universe of discourse, and let $S$ be a set of computer representations. For each $n=1,2, \ldots$, we define the notions of $n$ th order degree of certainty and of a $n$-th order fuzzy set, by the following induction over $n$ :

- By a first-order degree of certainty, we mean an element $s \in S$ (i.e., the set $S_{1}$ of all first-order degrees of certainty is exactly $S$ ).

- For every $n$, by a $n$-th order fuzzy set, we mean a function $\mu: U \rightarrow S_{n}$ from the universe of discourse $U$ to the set $S_{n}$ of all $n$-th order degrees of certainty.

- For every $n>1$, by a $n$-th order degree of certainty, we mean a function $s_{n}$ which maps every value $s \in S$ into an $(n-1)$-th order degree of certainty (i.e., a function $s_{n}: S \rightarrow S_{n-1}$ ).

Definition 4.5. Let $W$ be a set of words, let $\langle S,\|\|$.$\rangle be an uncertainty$ representation, and let $a$ be an adequacy function. For every $n>1$, and for every word $w \in W$, we define the $n$-th order degree of uncertainty $\|w\|_{a, n} \in S_{n}$ corresponding to the word $w$ as follows:

- As a first order degree of uncertainty $\|w\|_{a, 1}$ corresponding to the word $w$, we simply take $\|w\|_{a, 1}=\|w\|$.

- If we have already defined degrees of orders $1, \ldots, n-1$, then, as an $n$-th order degree of uncertainty $\|w\|_{a, n} \in S_{n}$ corresponding to the word $w$, we take a function $s_{n}$ which maps every value $s \in S$ into a $(n-1)$-th order degree $\|a(w, s)\|_{a, n-1}$.

Definition 4.6. Let $W$ be a set of words, let $\langle S,\|\|$.$\rangle be an uncertainty$ representation, let $a$ be an adequacy function, and let $P$ be a fuzzy property on a universe of discourse $P$. Then, by a $n$-th order fuzzy set (or a $n$-th order membership function) $\mu_{P, a}^{(n)}(x)$ corresponding to $P$, we mean a function which maps every value $x \in U$ into an $n$-th order degree of certainty $\|P(x)\|_{a, n}$ which corresponds to the word $P(x) \in W$. 
We will prove that for properties which are non-degenerate in some reasonable sense, it is sufficient to know the first and second order membership functions, and then the others can be uniquely reconstructed. Moreover, if we know the membership functions of first two orders for a non-degenerate class of fuzzy properties, then we will be able to reconstruct the higher order membership functions for all fuzzy properties from this class.

\section{Definition 4.7 .}

- We say that a fuzzy property $P$ on a universe of discourse $U$ is nondegenerate if for every $w \in W$, there exists an element $x \in U$ for which $P(x)=w$.

- We say that a class $\mathcal{P}$ of fuzzy properties $P$ on a universe of discourse $U$ is non-degenerate if for every $w \in W$, there exists a property $P \in \mathcal{P}$ and an element $x \in U$ for which $P(x)=w$.

Comment. For example, if $W \neq\{0,1\}$, then every crisp property, i.e., every property for which $P(x) \in\{0,1\}$ for all $x$, is not non-degenerate (i.e., degenerate).

Theorem 4.1. (Nguyen and Kreinovich $[21,39])$ Let $W$ be a set of words, let $\langle S,\|\|$.$\rangle be a sufficiently rich uncertainty representation, let U$ be a universe of discourse. Let $P$ and $P^{\prime}$ be fuzzy properties, so that $P$ is non-degenerate, and let $a$ and $a^{\prime}$ be adequacy functions. Then, from $\mu_{P, a}^{(1)}=\mu_{P^{\prime}, a^{\prime}}^{(1)}$ and $\mu_{P, a}^{(2)}=$ $\mu_{P^{\prime}, a^{\prime}}^{(2)}$, we can conclude that $\mu_{P, a}^{(n)}=\mu_{P^{\prime}, a^{\prime}}^{(n)}$ for all $n$.

\section{Comments.}

- In other words, under reasonable assumptions, for each property, the information contained in the first and second order fuzzy sets is sufficient to reconstruct all higher order fuzzy sets as well; therefore, in a computer representation, it is sufficient to keep only first and second order fuzzy sets.

- This result is somewhat similar to the well-known result that a Gaussian distribution can be uniquely determined by its moments of first and second orders, and all higher order moments can be uniquely reconstructed from the moments of the first two orders.

- It is possible to show that the non-degeneracy condition is needed, because if a property $P$ is not non-degenerate, then there exist adequacy functions $a \neq a^{\prime}$ for which $\mu_{P, a}^{(1)}=\mu_{P, a^{\prime}}^{(1)}$ and $\mu_{P, a}^{(2)}=\mu_{P, a^{\prime}}^{(2)}$, but $\mu_{P, a}^{(3)} \neq \mu_{P, a^{\prime}}^{(3)}$ already for $n=3$.

Theorem 4.2. (Nguyen and Kreinovich [21]) Let $W$ be a set of words, let $\langle S,\|\|$.$\rangle be a sufficiently rich uncertainty representation, let U$ be a universe of discourse. Let $\mathcal{P}$ and $\mathcal{P}^{\prime}$ be classes of fuzzy properties, so that the class $\mathcal{P}$ is non-degenerate, and let $\varphi: \mathcal{P} \rightarrow \mathcal{P}^{\prime}$ be a 1-1-transformation, and let $a$ and $a^{\prime}$ be adequacy functions. Then, if for every $P \in \mathcal{P}$, we have $\mu_{P, a}^{(1)}=\mu_{\varphi(P), a^{\prime}}^{(1)}$ and $\mu_{P, a}^{(2)}=\mu_{\varphi(P), a^{\prime}}^{(2)}$, we can conclude that $\mu_{P, a}^{(n)}=\mu_{\varphi(P), a^{\prime}}^{(n)}$ for all $n$. 
Comment. So, even if we do not know the adequacy function (and we do not know the corresponding fuzzy properties $P \in \mathcal{P}$ ), we can still uniquely reconstruct fuzzy sets of all orders which correspond to all fuzzy properties $P$.

\section{Operations Which are Consistent both with Granularity and Higher-Order Logics: Preliminary Results}

Why only unary and binary operations? Traditionally, in logic, only unary and binary operations are used as basic ones - e.g., "not", "and", "or" - while the only ternary (and higher order) operations are the operations which come from a combination of unary and binary ones.

A natural question is: are such combinations sufficient? I.e., to be more precise, can an arbitrary logical operation be represented as a combination of unary and binary ones?

For the classical logic, with the binary set of truth values $V=\{0,1\}$ $(=\{$ false, true $\})$, the positive answer to this question is well known. Indeed, it is known that an arbitrary logical operation $f: V^{n} \rightarrow V$ can be represented, e.g., in DNF form and thus, it can indeed be represented as a combination of unary ("not") and binary ("and" and "or") operations.

We are interested in explaining why unary and binary logical operations are the only basic ones. If we assume that the logic of human reasoning is the two-valued (classical) logic, then the possibility to transform every logical function into a DNF form explains this empirical fact.

In the traditional fuzzy logic, the set of truth values is the entire interval $V=[0,1]$. This interval has a natural notion of continuity, so it is natural to restrict ourselves to continuous unary and binary operations.

With this restriction in place, a natural question is: can an arbitrary continuous function $f:[0,1]^{n} \rightarrow[0,1]$ be represented as a composition of continuous unary and binary operations? The positive answer to this question was obtained in our papers $[38,42]$.

We have already mentioned, in the introduction, that the traditional fuzzy logic is not $100 \%$ adequate in describing expert reasoning, we need to modify it. In modifications motivated by granularity, we have a finite-valued logic $V$, in which $V$ is a finite set. In interval-values and other higher-order modifications, we get a multi- $D$ fuzzy logic.

In mathematical terms, $V$ be a closure of a simply connected bounded open set in $R^{m}, m>1$ (e.g., of a convex set). For example, for intervalvalued fuzzy sets,

$$
V=\{(a, b) \mid 0 \leq a \leq b \leq 1\} .
$$

Uncertainty of expert estimates is only one reason why we may want to go beyond the traditional $[0,1]$-valued logic; there are also other reasons: 
- A 1-D value is a reasonable way of describing the uncertainty of a single expert. However, the confidence strongly depends on the consensus between different experts. We may want to use additional dimensions to describe how many expert share the original expert's opinion, and to what degree; see, e.g., [25,43].

- Different experts may strongly disagree. To describe the degree of this disagreement, we also need additional numerical characteristics, which make the resulting logic multi-D; see, e.g., [41].

In all these cases, we need a multi-D logic to adequately describe expert's degree of confidence.

Natural questions are:

- Can every operation on a finite fuzzy logic be represented as a composition of unary and and binary operations?

- Can every (continuous) operation on a multi-D fuzzy logic be represented as a composition of (continuous) unary and and binary operations?

In this section, we show that both for finite-valued logics and for multi-D logics, every logical operation can be represented as a composition of unary and binary operations. Thus, we give a general explanation for the above empirical fact.

Theorem 5.1. (Nguyen, Kreinovich, and Goodman [40]) For every finite set $V$, and for every positive integer $n$, every $n$-ary operation $f: V^{n} \rightarrow V$ can be represented as a composition of unary and binary operations.

Theorem 5.2. (Nguyen, Kreinovich, and Goodman [40]) For every multi-D set of truth values $V$, and for every positive integer $n$, every continuous $n$ ary operation $f: V^{n} \rightarrow V$ can be represented as a composition of continuous unary and binary operations.

This result is based on the following known result:

Theorem. (Kolmogorov [20]) Every continuous function of three or more variables can be represented as a composition of continuous functions of one or two variables.

This result was proven by A. N. Kolmogorov as a solution to the conjecture of Hilbert, formulated as the thirteenth problem [16]: one of 22 problems that Hilbert has proposed in 1900 as a challenge to the 20 century mathematics.

This problem can be traced to the Babylonians, who found (see, e.g., [8]) that the solutions $x$ of quadratic equations $a x^{2}+b x+c=0$ (viewed as function of three variables $a, b$, and $c$ ) can be represented as superpositions of functions of one and two variables, namely, arithmetic operations and square roots. Much later, similar results were obtained for functions of five variables $a, b, c, d, e$, that represent the solution of quartic equations $a x^{4}+b x^{3}+c x^{2}+$ $d x+e=0$. But then, Galois proved in 1830 that for higher order equations, we cannot have such a representation. This negative result has caused Hilbert 
to conjecture that not all functions of several variables can be represented by functions of two or fewer variables. Hilbert's conjecture was refuted by Kolmogorov (see, e.g., [29], Chapter 11) and his student V. Arnold.

It is worth mentioning that Kolmogorov's result is not only of theoretical value: it was used to speed up actual computations (see, e.g., $[12,14,23,24,34,35])$.

It turns out that one can generalize Kolmogorov's theorem and prove that a similar representation holds for multi-D logics as well.

Conclusion. Traditionally, in logic, only unary and binary operations are used as basic ones. In traditional (2-valued) logic, the use of only unary and binary operations is justified by the known possibility to represent an arbitrary $n$-ary logical operation as a composition of unary and binary ones. A similar representation result is true for the $[0,1]$-based fuzzy logic. However, the $[0,1]$-based fuzzy logic is only an approximation to the actual human reasoning about uncertainty. A more accurate description of human reasoning requires that we take into consideration the uncertainty with which we know the values from the interval $[0,1]$. This additional uncertainty leads to two modifications of the $[0,1]$-based fuzzy logic: finite-valued logic and multi-D logic.

We show that for both modifications, an arbitrary $n$-ary logical operation can be represented as a composition of unary and binary ones. Thus, the above justification for using only unary and binary logical operation as basic ones is still valid if we take interval uncertainty into consideration.

\section{Conclusions}

Fuzzy logic is a natural way to incorporate expert knowledge into an intelligent system. Traditional [0,1]-based fuzzy logic has led to many successful applications, but in several aspects, the resulting computer representation is somewhat different from the original expert meaning. Two related approaches have been used to make fuzzy logic more adequate in representing expert reasoning: granularity and higher-order approaches. Each approach is successful in some applications where the other approach did not succeed so well; it is therefore desirable to combine these two approaches. This idea of combining the two approaches is very natural, but so far, it has led to few successful practical applications.

In this chapter, we provide results aimed at finding a better (ideally optimal) way of combining these approaches. Specifically:

- we show, on some reasonable examples, what is the optimal granularity;

- we handle the problem of selecting operations that are in optimal agreement with the granularity;

- we use the common origin of granularity and higher-order logics to find out which of the higher-order approaches is optimal; 
- finally, we provide preliminary results about operations which are consistent both with granularity and higher-order logics.

\section{Acknowledgments}

This work was supported in part by NASA under cooperative agreement NCC5-209 and grant NCC2-1232, by NSF grants CDA-9522207, ERA0112968 and 9710940 Mexico/Conacyt, by Future Aerospace Science and Technology Program (FAST) Center for Structural Integrity of Aerospace Systems, effort sponsored by the Air Force Office of Scientific Research, Air Force Materiel Command, USAF, under grants numbers F49620-95-1-0518 and F49620-00-1-0365, by Grant No. W-00016 from the U.S.-Czech Science and Technology Joint Fund, and by IEEE/ACM SC2001 Minority Serving Institutions Participation Grant.

The authors are thankful to Dr. Ajith Abraham and to the anonymous referees for valuable suggestions.

\section{References}

1. Agustií, J., et al. (1992) Structured local fuzzy logics in MILORD", In: Zadeh, L., Kacrpzyk, J., eds.: Fuzzy Logic for the Management of Uncertainty, Wiley, N.Y., 523-551.

2. Bandler, W., Kohout, L. J. (1984) Unified theory of multi-valued logical operations in the light of the checklist paradigm, Proc. of IEEE Conference on Systems, Man, and Cybernetics, Halifax, Nova Scotia, Oct. 1984, 356-364.

3. Barwise, J., ed. (1977) Handbook of Mathematical Logic, North-Holland, Amsterdam.

4. Beeson, M. J. (1985) Foundations of computable mathematics, Springer-Verlag, N.Y.

5. Ben-Or, N., Kozen, D., and Reif, J. (1986) The complexity of elementary algebra and geometry, Journal of Computer and System Sciences 32, 251-264.

6. Bishop, E. (1967) Foundations of Computable Analysis, McGraw-Hill.

7. Bishop, E., Bridges, D. S. (1985) Computable Analysis, Springer, N.Y.

8. Boyer, C. B., Merzbach, U. C. (1991) A History of Mathematics, Wiley, N.Y.

9. Bridges, D. S. (1979) Computable Functional Analysis, Pitman, London.

10. Canny, J. (1993) Improved algorithms for sign determination and existential quantifier elimination. The Computer Journal 36, 409-418.

11. Enderton, H. B. (1972) A Mathematical Introduction to Logic, Academic Press, N.Y.

12. Frisch, H. L., Borzi, C., Ord, G., Percus, J. K., Williams, G. O. (1989) Approximate Representation of Functions of Several Variables in Terms of Functions of One Variable, Physical Review Lett. 63(9), 927-929.

13. Godo, L., Lopez de Mantaras, R., Sierra, C., Verdaguer, A. (1989) MILORD: The Architecture and management of Linguistically expressed Uncertainty, International Journal of Intelligent Systems 4, 471-501. 
14. Hecht-Nielsen, R. (1987) Kolmogorov's Mapping Neural Network Existence Theorem, IEEE Int'l Conf. on Neural Networks, San Diego 2, 11-14.

15. Heindl, G., Kreinovich, V., Rifqi, M. (2002) In case of interval (or more general) uncertainty, no algorithm can choose the simplest representative, Reliable Computing 8, 213-227.

16. Hilbert, D. (1902) Mathematical Problems, lecture delivered before the Int'l Congress of Mathematics in Paris in 1900, translated in Bull. Amer. Math, Soc. 8, 437-479.

17. Hobbs, J. R. (2000) Half orders of magnitude, In: Obrst, L., Mani, I., eds.: Proc. of KR'2000 Workshop on Semantic Approximation, Granularity, and Vagueness, Breckenridge, Colorado, April 11, 28-38.

18. Hobbs, J. R., Kreinovich, V. (2001) Optimal Choice of Granularity In Commonsense Estimation: Why Half-Orders of Magnitude, Proc. IFSA/NAFIPS'2001, Vancouver, Canada, July 25-28, 1343-1348.

19. Klir, G., Yuan, B. (1995) Fuzzy Sets and Fuzzy Logic: Theory and Applications, Prentice Hall, Upper Saddle River, NJ.

20. Kolmogorov, A. N. (1957) On the Representation of Continuous Functions of Several Variables by Superposition of Continuous Functions of One Variable and Addition, Dokl. Akad. Nauk SSSR 114, 369-373.

21. Kreinovich, V., Nguyen, H. T. (2001) 1st Order, 2nd Order, What Next? Do We Really Need Third-Order Descriptions: A View From A Realistic (Granular) Viewpoint, Proc. IFSA/NAFIPS'2001, Vancouver, Canada, July 25-28, 19081913.

22. Kreinovich, V., Nguyen, H. T., Pedrycz, W. (2001) How to Make Sure That $\approx$ $100+1$ Is $\approx 100$ in Fuzzy Arithmetic: Solution and Its (Inevitable) Drawbacks, Proc. IFSA/NAFIPS'2001, Vancouver, Canada, July 25-28, 1653-1658.

23. Kurkova, V. (1991) Kolmogorov's Theorem Is Relevant, Neural Computation 3, 617-622.

24. Kurkova, V. (1992) Kolmogorov's Theorem and Multilayer Neural Networks, Neural Networks 5, 501-506.

25. Langrand, G., Kreinovich, V., Nguyen, H. T. (1995) Two-dimensional fuzzy logic for expert systems, Sixth International Fuzzy Systems Association World Congress, San Paulo, Brazil, July 22-28, 1 221-224.

26. Lewis, L. R., Papadimitriou, C. H. (1981) Elements of the theory of computation, Prentice-Hall, Englewood Cliffs, NJ.

27. Li, M., Vitányi, P. (1997) An Introduction to Kolmogorov Complexity and its Applications, Springer-Verlag, N.Y.

28. Lin, T.-Y., ed. (2002) Data Mining, Rough Sets and Granular Computing, Springer Verlag, Berlin.

29. Lorentz, G. G. (1966) Approximation of functions, Halt, Reinhart, and Winston, N.Y.

30. Martin, J. C. (1991) Introduction to Languages and the Theory of Computation, McGraw-Hill, N.Y.

31. McCarty, G. (1988) Topology, Dover, New York.

32. Mendel, J. (2001) Uncertain Rule-Based Fuzzy Logic Systems: Introduction and New Directions, Prentice-Hall, Upper Saddle River, NJ.

33. Proc. NAFIPS/IFIS/NASA'94, San Antonio, December 18-21, 1994.

34. Nakamura, M., Mines, R., Kreinovich, V. (1993) Guaranteed intervals for Kolmogorov's theorem (and their possible relation to neural networks), Interval Computations No. 3, 183-199. 
35. Nees, M. (1994) Approximative versions of Kolmogorov's superposition theorem, proved constructively, J. Comput. Appl. Math. 54(2), 239-250.

36. Nesterov, V. M. (1994) Interval analogues of Hilbert's 13th problem, Abstracts of the Int'l Conference Interval'94, St. Petersburg, Russia, March 7-10, 185186.

37. Nguyen, H. T., Kreinovich, V. (1995) Towards theoretical foundations of soft computing applications, Int'l J. on Uncertainty, Fuzziness, and KnowledgeBased Systems 3(3), 341-373.

38. Nguyen, H. T., and Kreinovich, V. (1997) "Kolmogorov's Theorem and its impact on soft computing", In: Yager, R.E., Kacprzyk, J.: The Ordered Weighted Averaging Operators: Theory and Applications, Kluwer, Boston, MA, 3-17.

39. Nguyen, H. T., Kreinovich, V. (1998) Possible new directions in mathematical foundations of fuzzy technology: a contribution to the mathematics of fuzzy theory, In: Nguyen Hoang Phuong and A. Ohsato, eds.: Proceedings of the Vietnam-Japan Bilateral Symposium on Fuzzy Systems and Applications VJFUZZY'98, HaLong Bay, Vietnam, 30th September-2nd October, 9-32.

40. Nguyen, H. T., Kreinovich, V., Goodman, I. R. (2001) Why Unary and Binary Operations in Logic: General Result Motivated by Interval-Valued Logics, Proc. IFSA/NAFIPS'2001, Vancouver, Canada, July 25-28, 1991-1996.

41. Nguyen, H. T., Kreinovich, V., Shekhter, V. (1998) On the Possibility of Using Complex Values in Fuzzy Logic For Representing Inconsistencies, International Journal of Intelligent Systems 13(8), 683-714.

42. Nguyen, H. T., Kreinovich, V., Sprecher, D. (1996) Normal forms for fuzzy logic - an application of Kolmogorov's theorem, International Journal on Uncertainty, Fuzziness, and Knowledge-Based Systems 4(4), 331-349.

43. Nguyen, H. T., Kreinovich, V., Wu, B. (1999) Fuzzy/probability frac$\mathrm{tal} / \mathrm{smooth}$, International Journal of Uncertainty, Fuzziness, and KnowledgeBased Systems (IJUFKS) 7(4), 363-370.

44. Nguyen, H. T., Kreinovich, V., Zuo, Q. (1997) Interval-valued degrees of belief: applications of interval computations to expert systems and intelligent control, International Journal of Uncertainty, Fuzziness, and Knowledge-Based Systems (IJUFKS) 5(3) 317-358.

45. Nguyen, H. T., Walker, E. A. (1999) First Course in Fuzzy Logic, CRC Press, Boca Raton, FL.

46. Papadimitriou, C. H. (1994) Computational Complexity, Addison Wesley, San Diego.

47. Puyol-Gruart, J. Godo, L., Sierra, C. (1992) A specialization calculus to improve expert systems communication, Research Report IIIA 92/8, Institut d'Investigació en Intelligènicia Artificial, Spain.

48. Schoenfield, J. R. (1967) Mathematical Logic, Addison-Wesley.

49. Seidenberg, A. (1954) A new decision method for elementary algebra. Annals of Math. 60 365-374.

50. Shafer, G., Pearl, J., eds. (1990) Readings in Uncertain Reasoning, M. Kaufmann, San Mateo, CA.

51. Tarski, A. (1948) A Decision Method for Elementary Algebra and Geometry, University of California Press, Berkeley.

52. Türkşen, I. B. (1986) Interval valued fuzzy sets based on normal forms, Fuzzy Sets and Systems 20, 191-210.

53. Wadsworth, H. M., ed. (1990) Handbook of Statistical Methods for Engineers and Scientists, McGraw-Hill Publishing Co., N.Y. 
54. Yamakawa, T., Kreinovich, V. (1999) Why Fundamental Physical Equations Are of Second Order?, International Journal of Theoretical Physics 38, 17631770 .

55. Zadeh, L. A. (1965) Fuzzy Sets, Information and Control 8, 338-353.

56. Zadeh, L. A. (1979) Fuzzy sets and information granularity, In: Gupta, M., Ragade, R., Yager, R. (eds.), Advances in Fuzzy Set Theory and Application, North Holland, Amsterdam, 3-18.

57. Zemanian, A. H. (1987) Distribution Theory and Transform Analysis, Dover, New York. 